\title{
Entre la crítica, la autonomía y la indiferencia: la población sin religión en México
}

\author{
Carlos Nazario Mora Duro*
}

\section{Resumen:}

Este artículo se pregunta, ¿cuáles son las principales características de la población sin religión en el contexto mexicano? Partiendo de la información de que este sector del campo religioso se ha incrementado visiblemente sobre todo desde las últimas décadas del siglo pasado, conformando un sector de alrededor de 5.3 millones de personas o, en otras palabras, $4.7 \%$ de la población total mexicana. Entonces, para describir las principales características del grupo sin religión se desarrollan tres apartados: primero, la conceptuación de la población desafiliada; segundo, la exposición de diversos datos representativos para conocer los atributos comparativos de este conjunto que no pertenece a una religión, entre ellos, las creencias y prácticas. Y, por último, a manera de discusión, se presenta una conjetura sobre las actitudes componente de esta categoría: la crítica, la autonomía y la indiferencia.

Palabras clave: sin religión, desafiliación religiosa, prácticas, creencias, secularización, México.

\section{Abstract:}

This papers analyzes what are the main characteristics of the non religious population in the Mexican context? Based on the information that, this sector of the religious field has increased especially since the last decades of the last century, forming a group of around 5.3 million people or, in other words, $4.7 \%$ of the total Mexican population. To describe the main characteristics of the religiously unaffiliated group, the paper begins by the conceptualization of non religious persons; secondly, it is exposes representative data to know the comparative attributes of this group of "nones", including beliefs and practices. And, finally, to discuss, it is presented a hypothesis about the attitudes component of this religious field category: criticism, autonomy and indifference.

Keywords: non religious persons, religiously unaffiliated, practices, beliefs, secularization, Mexico.

* Doctor en Ciencia Social con especialidad en Sociología por El Colegio de México (COLMEX). Profesor del Centro de Estudios Antropológicos de la Facultad de Ciencias Políticas y Sociales de la Universidad Nacional Autónoma de México (UNAM). Líneas de investigación: sociología de la religión, sociología cultural, secularización y laicidad, redes virtuales de información y comunicación. Correo: cmora@colmex.mx. 


\section{Introducción}

México es reconocido como un país tradicionalmente católico y con un fervor significativo hacia manifestaciones religiosas como el caso de la Virgen de Guadalupe. No es extraño, en esta lógica, que $83 \%$ de mexicanos afirmen su fe hacia la Guadalupana y dentro de los católicos esta proporción aumente hasta $95 \%$ de ellos (Católicas por el Derecho a Decidir, 2014; Parametría, 2015).

A pesar de esta imagen religiosa asociada al nacionalismo mexicano (Lafaye, 2015), los cambios socioculturales de la segunda mitad del siglo XX y principios del siglo XXI, han mostrado que el país se ha diversificado en términos religiosos y ha pluralizado el mercado de los bienes de salvación (Bastian, 2013, p. 94). En consecuencia, el campo religioso mexicano contemporáneo está configurado por tres tendencias destacables:

La primera de ellas es un decrecimiento del catolicismo, que a principios del siglo XX agrupaba $99 \%$ de las afiliaciones, pero que, en la actualidad, ha disminuido su proporción a alrededor de $80 \%$ de identificaciones religiosas (INEGI, 2005, 2010, 2011). ${ }^{1}$ Por otro lado, también se ha observado el incremento del cristianismo no católico y la apertura de los mexicanos hacia otras opciones religiosas. Esto ha provocado que, hoy en día, casi $10 \%$ de la población nacional practique una religión distinta al catolicismo, sobre todo aquellas de corte bíblico o evangélicas. Y, en tercer lugar, el ensanchamiento de la población sin religión o, en otras palabras, ese sector que ya no se identifica con alguna de las opciones del campo religioso. Este grupo, complemento de los conjuntos religiosos, de acuerdo con el censo nacional mexicano de 2010 , constituye $4.7 \%$ de la población mexicana o, en números concretos, alrededor de 5.3 millones de personas.

A partir de su creciente escala estadística, es importante preguntarse, ¿cuáles son las principales características de la población sin religión en el contexto mexicano? Así planteado, este artículo tiene el objetivo de describir las principales tendencias del grupo sin religión y para ello se desarrollan tres apartados. En el primero, se aborda la conceptuación de la población sin religión; en el segundo, se detallan diversos datos representativos para conocer los atributos comparativos de este sector poblacional en México.

Esta primera transformación del campo religioso mexicano coloca al país en el quinto puesto dentro de las naciones con más porcentaje de catolicismo, debajo de Paraguay (88 \%), Ecuador (81\%), Venezuela $(79 \%$ ) y Perú $(77 \%$ ) (Latinobarómetro, 2014). 
Y, por último, a manera de discusión, se presenta una conjetura sobre las actitudes componente de este conjunto del campo religioso.

La hipótesis ulterior es que existen tres posiciones principales dentro de este conjunto heterogéneo denominado sin religión:

La primera de ellas es una actitud de autonomía, cuya principal postura puede ser encontrada entre los "creyentes sin iglesia" o aquellos denominados "espirituales, pero no religiosos", es decir, personas que gestionan individualmente los bienes de salvación alejados de cualquier autoridad institucional eclesiástica. La segunda es una actitud crítica alrededor del espectro religioso, por supuesto, expresada en perfiles como los ateos, quienes suelen cuestionar no solamente la estructura institucional sino también el dogma profesado por los diversos credos religiosos. $\mathrm{Y}$, finalmente, una actitud de indiferencia, como en el caso de los agnósticos, quienes más que gestionar individualmente su propia creencia y práctica religiosa o controvertir los dogmas de la cosmovisión religiosa, consideran que el tópico carece de peso significativo en su vida cotidiana o que representa un universo de conceptos sin trascendencia simbólica.

\section{El concepto de población sin religión}

Desde las últimas décadas del siglo XX se ha abordado el fenómeno de las personas sin religión sobre todo desde la literatura anglosajona y, particularmente, en el caso de Estados Unidos, donde la categoría se asoció a las transiciones generales de la secularización, es decir, "al concepto multifacético que connota el declive de la significancia social de la religión" y donde uno de sus aspectos fundamentales concierne a la prevalencia de las tradiciones religiosas $y$, por tanto, a la evidencia del incremento de personas que no declaran ninguna preferencia religiosa (Condran \& Tamney, 1985, p. 415).

Ya desde el periodo aludido, los sin religión -también definidos como desafiliados religiosos o nones- fueron descritos como ateos, agnósticos o personas que no tienen religión. Y, además, se estableció que el incremento de la frecuencia de nones, no significaba necesariamente que las personas eran menos religiosas, sin embargo, tal cambio sí sugería que las tradiciones religiosas existentes habían devenido "menos relevantes" en el ámbito social (Condran \& Tamney, 1985, pp. 415-416).

En el caso latinoamericano, el trabajo de Regina Gibaja sobre las actitudes seculares de obreros y campesinos urbanos y rurales de Argentina, también se sumó a las investigaciones interesadas en conocer el cambio de 
actitudes religiosas de la población, bajo el supuesto de que los individuos más "expuestos a la influencia de la educación y la vida urbana", tendrían una actitud más orientada secularmente y menos religiosa, al mismo tiempo que disminuían la frecuencia de prácticas y lazos con organizaciones religiosas, y exhibían más tolerancia religiosa que otros sectores sociales (Gibaja, 1972, pp. 196-197).

En el trabajo citado, Gibaja no delimitó un concepto específico de la población sin religión debido a que para esos años en Argentina el número de personas sin afiliación religiosa (o, incluso, con una afiliación diferente a la católica) era muy bajo como para "considerarlo separadamente". En sus palabras, "la afiliación religiosa tiene menos importancia que el conjunto de actitudes y creencias de los entrevistados sobre lo sagrado y su incidencia en la vida secular" (Gibaja, 1972, p. 201).

Por su parte, para el contexto mexicano, el crecimiento de la desafiliación religiosa, sobre todo en las últimas décadas del siglo XX, puso en la agenda de investigación a la población sin religión, partiendo de su definición en el censo nacional. De esta manera, hasta el año 2000, de acuerdo con el catálogo del Instituto Nacional de Estadística y Geografía (INEGI), el censo agrupó en el conjunto sin religión a quien expresó ser ateo, escéptico, agnóstico, no religioso o no tener ninguna religión en particular.

Uno de los documentos que consideró y analizó los datos generados por el registro nacional de 2000 fue el Atlas de la diversidad religiosa en México (de la Torre y Gutiérrez, 2007); y en él se especificó que las personas sin religión son aquellos que poseen:

...desde la cosmovisión de la inexistencia de un dios (ateísmo), la convicción en la imposibilidad de afirmar o negar la existencia de un dios (agnosticismo), o la postura de quien independientemente de sus creencias no se considera miembro de ninguna institución religiosa (escepticismo, antidogmatismo, práctica de "el costumbre", búsqueda espiritual sin pertenencia religiosa institucional). Adicionalmente, es probable que esta categoría agrupe también a aquellos insatisfechos con las opciones censales dadas a la pregunta sobre pertenencia religiosa, o aquellos que no desean dar a conocer su adscripción (Gutiérrez, 2007, p. 116).

Por otro lado, para 2010, el mismo padrón nacional registró una delimitación adicional. Consideró que las personas sin religión son una población que "no tiene preferencia por alguna religión" o que adopta una posición, 
“ante la existencia de un dios, ser o poder supremo", con alguna de las siguientes orientaciones:

- Dogmática. Qué afirma la inexistencia de un dios

- Escéptica. Que declara incapacidad del hombre para comprender un concepto supremo

- Crítica. Que considera las pruebas del teísmo insuficientes

- Práctica. Que muestra indiferencia ante la existencia o inexistencia de un ser o poder superior

De herejía. En oposición al dogma de una iglesia particular

A lo anterior, se suma, "aquella población creyente de una religión de contenido esencial filosófico, que considera que más que una religión, su práctica es una forma de vida y por tanto como religión no se declaran partidarios." (INEGI, 2015, p. 8).

Hasta este punto, las definiciones mentadas, dan cuenta de once subcategorías que complejizan, de primera mano, una delimitación homogénea del concepto sin religión (Tabla 1).

\begin{tabular}{|l|}
\hline \multicolumn{1}{|c|}{ Tabla 1. Subcategorías sin religión } \\
\hline 1. Ateo \\
\hline 2. Escéptico \\
\hline 3. Agnóstico \\
\hline 4. No religioso \\
\hline 5. Sin preferencia religiosa \\
\hline 6. No tiene religión \\
\hline 7. Crítico \\
\hline 8. Hereje \\
\hline 9. Costumbres indígenas \\
\hline 10. Buscador espiritual \\
\hline 11. Insatisfechos \\
\hline
\end{tabular}

Elaboración propia.

Debido a la complejidad expuesta, parece importante plantear una definición que hemos propuesto en la tesis doctoral Creer sin iglesia y practicar sin Dios: población sin religión en el contexto urbano y rural de México en los albores del siglo XXI (Mora, 2017). 
En este trabajo, se retoma el debate de la secularización, considerando sus procesos generales como heterogéneos e híbridos, y en relación con los contextos locales y nacionales, aunque, conservando el argumento de que existe una disminución de la influencia de la esfera religiosa en la sociedad contemporánea, sin que esto implique la supresión de la religión, la religiosidad o lo religioso de los espacios públicos y privados de la sociedad, antes bien, involucra la "reorganización permanente del trabajo de la religión" (Hervieu-Léger, 1985), es decir, el "movimiento constante -en el propio individuo- de la afiliación, las creencias, las prácticas y, por tanto, de las experiencias en torno a lo profano y lo sagrado" (Mora, 2017, p. 20).

A partir de lo anterior, se define a la población sin religión como un conjunto de personas que manifiesta una desafiliación respecto a una o varias de las tradiciones del campo religioso, sin que esta desafiliación inicial corresponda intrínsecamente con una prescindencia absoluta de creencias y de prácticas de carácter religioso o espiritual.

La desafiliación religiosa, entonces, puede manifestarse como una falta de identificación o, incluso, como un tipo de aversión directa hacia un "linaje creyente", ya que la persona expresa una falta de continuidad respeto a "una comunidad que reúne a los creyentes pasados, presentes y futuros" (Hervieu-Léger, 1996, p. 28). ${ }^{2}$ Sin embargo, en términos de las creencias y prácticas, los tipos ideales normativos (por ejemplo el ateo que no cree y no practica) son poco recurrentes entre las personas sin religión y, más bien, es altamente probable encontrar una diversificación de perfiles religiosos en movimiento como parte de un fenómeno de secularización individual o de secularización de la conciencia (Mora, 2017, p. 274).

Esta propuesta, sin duda, tiene que respaldarse con los datos y atributos específicos del grupo sin religión. Por lo cual la siguiente parte de este artículo se ocupa de presentar las principales características de los desafiliados mexicanos.

\section{Características de la población sin religión en México}

Ya se ha mencionado que, en términos de porcentajes, los sin religión en México representan $4.7 \%$ de la población total del país, esto es, alrededor

2 Usando el vocabulario de Hervieu-Léger (1996), se ha definido que el linaje creyente comprende, por un lado, a la comunidad de creyentes concretos de una iglesia (un grupo social específico con formas de organización diversas, formales e informales) $y$, al mismo tiempo, una comunidad virtual, a la manera de "linaje imaginario" (pasado y futuro), que sirve para identificar a los que "están dentro" y "los que están fuera" de ella (Mora, 2017, p. 67). 
de 5.3 millones de mexicanos. De esta información se obtiene que, por cada 100 católicos en México hay 6 personas sin religión y 12 con otra adscripción religiosa (Tabla 2).

Tabla 2. Porcentaje de grupos religiosos en México y razón con base en el catolicismo

\begin{tabular}{|l|c|c|c|}
\hline Grupo religioso & Porcentaje & Razón (base \% católicos) & Razón * 100 \\
\hline Católicos & 82.7 & 1 & 100 \\
\hline Otra religión & 9.9 & 0.12 & 12 \\
\hline Sin religión & 4.7 & 0.06 & 6 \\
\hline
\end{tabular}

Elaboración propia. Fuente: INEGI (2010). Nota: otra religión incluye a protestantes y evangélicas, bíblicas diferentes de evangélicas, judaica y otras religiones. No se incluye el porcentaje de no especificado.

\subsection{Desarrollo histórico}

La franja de personas que no comulgan con una religión en particular tiene presencia de larga data en México, al menos en los resultados censales registrados desde finales del siglo XIX. En el primer censo nacional realizado en 1895, $99.1 \%$ de la población se declaró católica, mientras que el segundo grupo más amplió fue el de la población sin religión (0.5\%). A partir de esta exigua presencia, la población sin religión ha mostrado dos picos de crecimiento marcado: el primero de ellos, en la etapa post revolucionaria, después de 1921; y, el segundo, en las últimas tres décadas del siglo XX (Gráfica 1).

Gráfica 1. Población sin religión en México, 1895-2010 (\%)

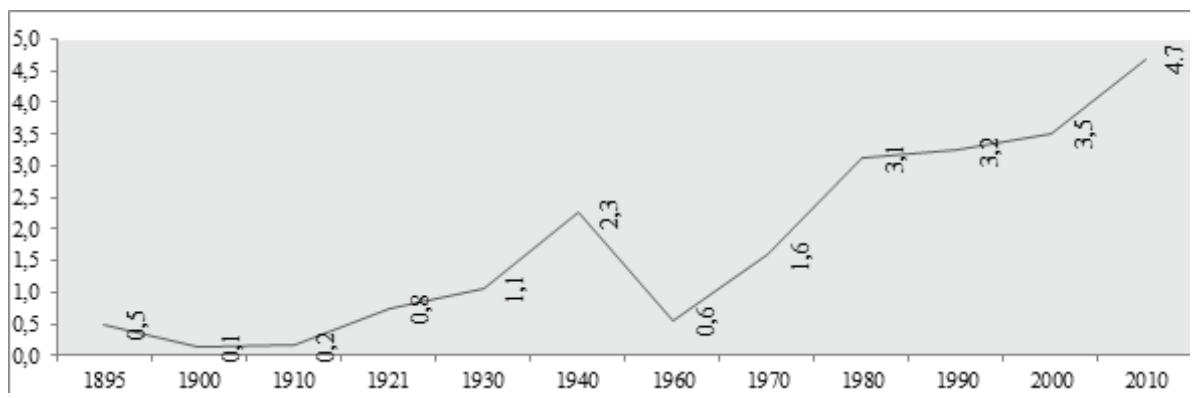

Elaboración propia. Fuente: INEGI (2010, 2011). Nota: En 1950 no se recogió la categoría sin religión. A partir de 1990 el universo de población es de 5 años y más, anteriormente era la población total. 
No es el objetivo de este documento analizar la tendencia histórica de éste y otros grupos del campo religioso, sin embargo, una relación general encontrada, con base en el desarrollo histórico y la distribución espacial de la desafiliación, es el incremento del conjunto sin religión a medida que la población católica disminuye y viceversa: allí donde el catolicismo es mayoritario las personas sin religión tienden a ser un grupo más pequeño.

\subsection{Perfil sociodemográfico}

Ahora bien, ¿qué se sabe del perfil de los desafiliados en el contexto mexicano? El censo nacional también aporta luz sobre esta interrogante. Para el año 2010, la población sin religión se constituyó $57.4 \%$ por hombres y $42.6 \%$ de mujeres (INEGI, 2011, p. 10). Esto implica que por cada 10 hombres que se declaran sin religión hay 7 mujeres desafiliadas. Esta mayoría masculina en el sector sin religión se ha observado en diversas encuestas, a diferencia del resto de los grupos religiosos en donde las mujeres muestran los mayores porcentajes. Este fenómeno, de hecho, ha generado una discusión particular sobre la inclinación marcada de las mujeres hacia el espectro religioso, por ejemplo, el libro Why are Women More Religious Than Men? (Trzebiatowska \& Bruce, 2012).

Por otro lado, la población sin religión en México se caracteriza también por ser un conjunto relativamente joven. Según datos del censo nacional, más de $60 \%$ de desafiliados se ubicó entre los primeros seis quintiles de edad, es decir, son personas menores de 34 años (para 2014) y, por lo tanto, representan generaciones que nacieron después de 1980, periodo en que el catolicismo mostró un declive significativo a nivel nacional. Este dato también está corroborado por el Pew Research Center en su trabajo Religión en América Latina. Cambio generalizado en una región históricamente católica (2014). En este documento, 1 de cada 3 personas sin religión en México se encontró entre los 18 y 34 años (Gráfica 2). 
Gráfica 2. Grupos religiosos e intervalos de edad en México, 2013

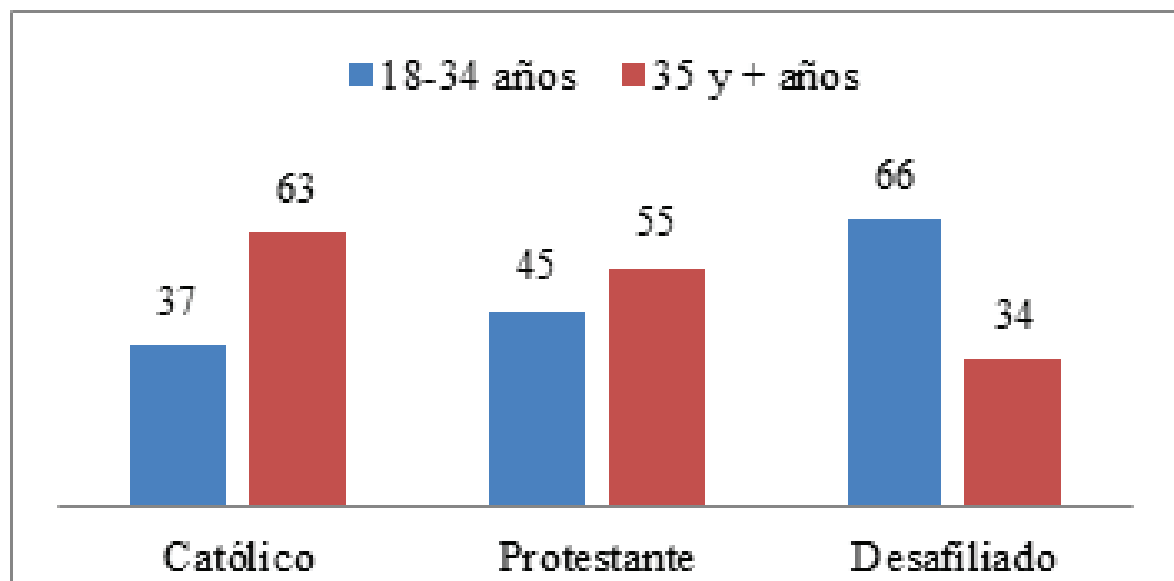

Elaboración propia. Fuente: Pew Research Center (2014).

Al considerar la variable educativa, las personas sin religión muestran niveles muy similares a la media nacional y a los de los católicos ( 9 años de escolaridad promedio), sin embargo, estudian menos años que los judíos (12 años) y la Iglesia de Jesucristo de los santos de los últimos días (11 años), también conocidos como mormones (Tabla 3).

\begin{tabular}{|l|c|c|c|}
\hline \multicolumn{4}{|c|}{$\begin{array}{c}\text { Tabla 3. Características educativas por grupo religioso } \\
\text { en México, 2010 (15 años y +) }\end{array}$} \\
\hline Grupo religioso & $\begin{array}{c}\text { \% saben leer } \\
\text { y escribir }\end{array}$ & $\begin{array}{c}\text { Promedio de años } \\
\text { de escolaridad }\end{array}$ & $\begin{array}{c}\text { \% sin concluir } \\
\text { educación básica }\end{array}$ \\
\hline Nacional & 92 & 9 & 42.6 \\
\hline Católicos & 93 & 9 & 42.5 \\
\hline $\begin{array}{l}\text { Protestante/ Pentecostal/ } \\
\text { Evangélica/ Cristiana }\end{array}$ & 92 & 8 & 44.6 \\
\hline Adventistas del Séptimo Día & 87 & 7 & 56.1 \\
\hline $\begin{array}{l}\text { Iglesia de Jesucristo de los } \\
\text { Santos de los Últimos Días }\end{array}$ & 97 & 11 & 24.7 \\
\hline Testigos de Jehová & 95 & 8 & 46.3 \\
\hline Judíos & 96 & 12 & 21.6 \\
\hline Sin religión & 92 & 9 & 40.8 \\
\hline
\end{tabular}

Elaboración propia. Fuente: INEGI (2011). 
La proporción de personas que sabe leer y escribir tampoco está por encima de la media nacional en el caso de los sin religión, aunque si tienen un porcentaje poco menor de personas sin concluir educación básica respecto del promedio del país (Tabla 3). Con base en lo anterior, no se puede aseverar, que hay una relación directa entre más educación y desafiliación religiosa en el caso mexicano. De hecho, en este mismo aspecto, la Encuesta Nacional sobre Creencias y Prácticas Religiosas en México (ENCREER) realizada en 2016 por la Red de Investigadores del Fenómeno Religioso en México (RIFREM), presenta que, dentro de los distintos grupos identificados por la medición, los sin religión son el conjunto con mayor porcentaje de personas sin estudios $(7.3 \%)$ y, al mismo tiempo, con la mayor proporción de individuos con educación superior $(22.8 \%)$.

Adicionalmente, la información sociodemográfica censal también muestra que los desafiliados son el conjunto del campo religioso con mayor porcentaje (61\%) de población económicamente activa (INEGI, 2011, p. 10). Esto, sin duda, puede relacionarse con la mayoría del sector joven que integra este conjunto, de tal manera que, al interior de los sin religión los porcentajes de "empleados", "trabajadores independientes" y "patrones" o "empresarios" son más altos que en otros grupos religiosos; y, en contraste, las "amas de casa" y los "jubilados o pensionados" son subconjuntos con las proporciones más bajas que en otros agregados religiosos (RIFREM, 2016).

Por último, dentro de los datos sociodemográficos, la categoría también exhibe que $22.8 \%$ de personas sin religión viven en comunidades con menos de 2500 habitantes. Y, además, del total de los desafiliados (de 3 años y más), 366483 declararon ser hablantes de una lengua indígena (INEGI, 2011). Esta última cifra equivale a $7 \%$ de la población total sin religión para 2010, lo que muestra que no es la categoría del campo religioso con más proporción de indígenas (hablantes). ${ }^{3}$

\footnotetext{
La proporción de indígenas hablantes y de la población en comunidades con pocos habitantes (menos de 2500 personas), puede dar cuenta del carácter urbano o rural de los grupos religiosos. Por ejemplo, los Adventistas del Séptimo Día se caracterizan por ser una categoría religiosa con cierta preeminencia de la variable rural-indígena ya que $50.6 \%$ de su población se encuentra en comunidades pequeñas y, además, el 17.2\% de ellos se declara hablante indígena. En contraste, los judíos son una religión con un carácter más urbano: solo $11.3 \%$ de ellos se concentra en poblaciones de menos de 2500 habitantes, mientras que $3.5 \%$ se identifica como hablante de una lengua indígena.
} 
Estos datos sugieren que la desafiliación comienza a concentrarse en poblaciones y aglomeraciones cada vez más urbanas y entre personas sin una identidad exclusivamente indígena. Para estos últimos, sin embargo, parece válida la conjetura de que la desafiliación podría "esconder" "otras religiones" bajo el ítem "ninguna" o el propio "catolicismo de la costumbre" que escapa a las regulaciones religiosas de la institución católica, más que "un crecimiento del ateísmo en un estado predominantemente rural" (Bastian, 2008, pp. 20-21); a pesar de lo anterior, tampoco puede descartarse que en las comunidades rurales los jóvenes con acceso a grados de educación por encima de la media comienzan a cuestionar los argumentos tradicionales desde los discursos seculares incorporados por su formación académica (Mora, 2017, p. 249).

Hasta este primer apartado se sabe que la población sin religión en México, en general, muestra un perfil de individuos jóvenes, nacidos sobre todo en los años ochenta y noventa del siglo pasado. Asimismo, la categoría tiene cierta mayoría masculina, a diferencia de todos los otros grupos religiosos donde las mujeres habitualmente sobrepasan el $50 \%$. Por su parte, la educación de esta población no está por encima de la media nacional, pese a ello, la frecuencia relativa suele ser más alta tanto para educación superior como para individuos sin estudios. Y, finalmente, hay un porcentaje considerable de población indígena y rural entre los desafiliados, sin embargo, no se puede caracterizar a esta categoría dentro de los grupos con más población en estas condiciones sociodemográficas.

\subsection{Prácticas y creencias entre los desafiliados}

Dos de las dimensiones más importantes al operacionalizar el tema religioso son las prácticas y creencias dentro de este espectro. Por supuesto, esto tiene sentido cuando se está definiendo a poblaciones religiosas en algún sentido, comenzando por su propia identificación dentro de un linaje creyente, aunque parece ser irrelevante cuando se analiza a la población sin religión.

No hay ninguna contradicción en preguntarse sobre las creencias y prácticas religiosas entre las personas sin religión, si recordamos que la secularización presupone una reorganización constante de las dimensiones del fenómeno religioso, de tal manera que, la desafiliación es coherente y concurrente con ciertas acciones y representaciones identificadas como religiosas o espirituales. 
Para encontrar las variables pertinentes, el censo nacional es una herramienta limitada y se debe echar mano de los resultados de encuestas que han profundizado en el tema de prácticas y creencias religiosas.

En esta lógica, el trabajo realizado por el Pew Research Center en 2014 ha encontrado que en México los desafiliados se caracterizan por ser un conjunto de creencias menos recurrentes que los otros grupos religiosos, aunque no hay evidencia que sustente la afirmación categórica de que las personas sin religión dejan de creer por el hecho de mostrar oposición hacia uno de los linajes o tradiciones del campo religioso (Gráfica 3).

Gráfica 3. Porcentaje de creencias afirmativas entre Católicos, Protestantes y Desafiliados en México, 2014

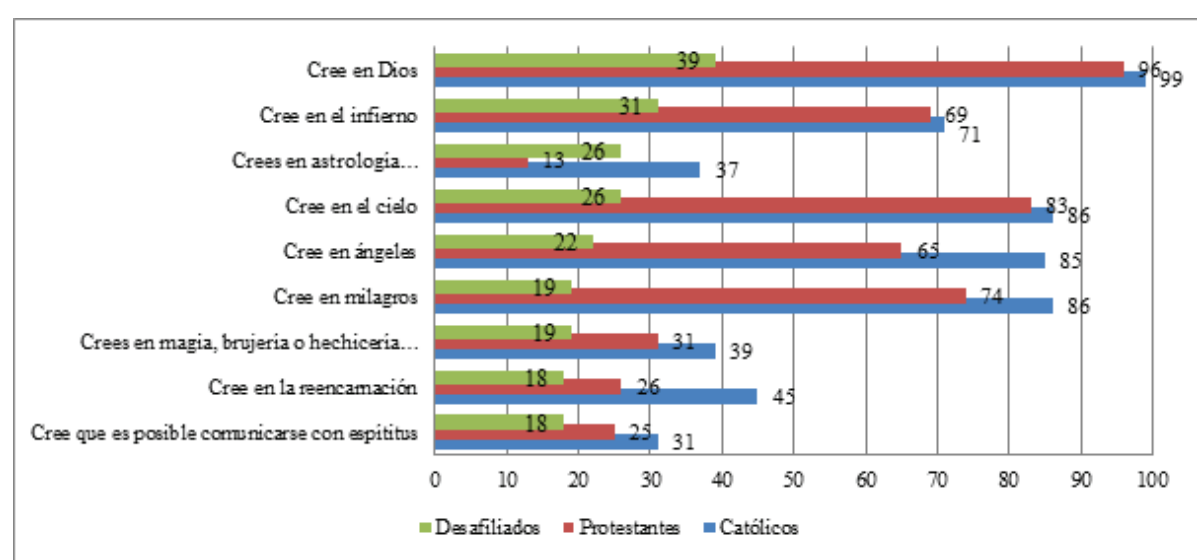

Elaboración propia. Fuente: Pew Research Center (2014).

De acuerdo con los datos obtenidos, la creencia en Dios es una de las principales representaciones entre los grupos religiosos en México, sin embargo, esta inclinación se atenúa entre las personas sin religión: alrededor de 4 de cada 10 desafiliados expresa una afirmación positiva sobre la creencia en una divinidad. ${ }^{4}$ Esta frecuencia equivale a una relación de momios

Sobre este punto, resalta que incluso los ateos declarados pueden conservar una creencia significativa en Dios. A decir de la literatura, en el caso de ateos declarados y militantes el proceso de desposesión de los elementos religiosos se desarrolla en distintas etapas: acercamiento, cuestionamiento y ambigüedad. Y, en algunos casos, el declararse como ateo, no implica que se carezca de creencias religiosas sustantivas. Ya diversas investigaciones han comentado que los ateos "también son creyentes" (Smith, 2010; LeDrew, 2013, 2014). 
particular: por cada 10 personas sin religión que asegura no creer en Dios, hay 6 de ellas que sí confirma su creencia; por su parte, entre los católicos, esta relación es de 10 / 990; y, entre protestantes de $10 / 240$.

Como puede observarse también en la Gráfica 3, todas las creencias ulteriores se ven disminuidas no solamente entre las personas sin religión, sino en el grueso de los conjuntos religiosos. En este sentido, en el orden de magnitud para los desafiliados -después de Dios- la representación más afirmada es el infierno (31\%), la creencia en la astrología (26\%), en el cielo (26\%), en los ángeles (22\%), los milagros (19\%), la magia (brujería o hechicería) $(19 \%)$, la reencarnación $(18 \%$ ) y la comunicación con espíritus $(18 \%)$.

Ahora bien, observando las prácticas religiosas de los desafiliados mexicanos, en primer lugar, se ha encontrado que determinarse sin religión tiene una considerable relación con una ausencia prolongada a servicios de tipo religioso. En la encuesta del Pew Research Center (2014), por ejemplo, 81 $\%$ de personas sin religión aseguró que "nunca" asiste a servicios religiosos, "además de bodas y funerales". Por su parte, solo $7 \%$ de católicos y $3 \%$ de protestantes aseguraron esta misma respuesta. Una tendencia similar se halló en la encuesta ENCREER (RIFREM, 2016), ya que 75 \% de los que aseguraron no pertenecer a una religión declararon nunca asistir a "servicios religiosos, misa o culto", mientras que solo $3.2 \%$ de los que pertenecen a una religión "nunca" asiste.

Respecto a la oración en el ámbito privado o fuera de servicios religiosos colectivos, la información también muestra que los desafiliados rara vez $(21 \%)$ o nunca $(62 \%)$ realizan este tipo de acciones identificadas como religiosas. Entre los católicos, este porcentaje agrupado (rara vez + nunca) de personas que no realizan oración es de $19 \%$ y entre protestantes solo $10 \%$.

A pesar de esta relativa disminución de prácticas específicas entre las personas desafiliadas, también es cierto que no existe una prescindencia absoluta de acciones rituales, religiosas o espirituales, aunque, sin duda hay una atenuación de la participación efectiva. En concreto, si se consideran otras indicadores de la dimensión práctica, además de la asistencia a servicios religiosos y la oración individual, los desafiliados también exponen frecuencias relativas por debajo de aquellos que pertenecen a una religión (Gráfica 4). 
Gráfica 4. Porcentaje de prácticas afirmativas entre personas que pertenecen y no a una religión en México, 2016

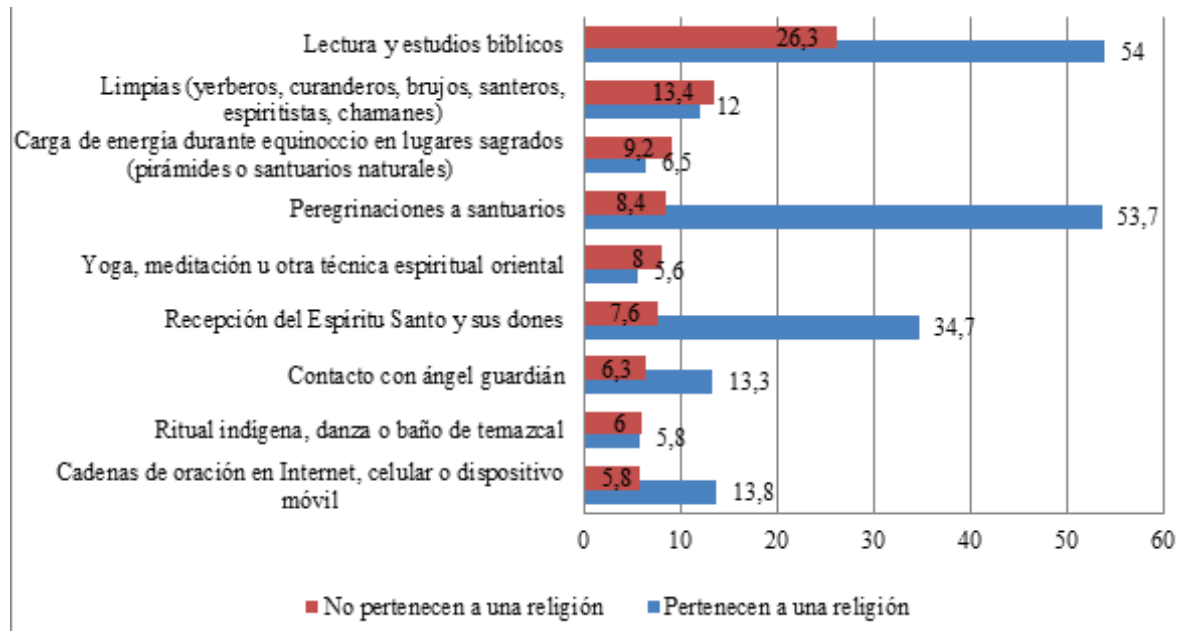

Elaboración propia. Fuente: ENCREER (RIFREM, 2016).

Con base en lo anterior, la ENCREER (RIFREM, 2016), presenta que las personas que no pertenecen a una religión exhiben porcentajes más bajos de acciones religiosas en los distintos rubros medidos, excepto en aquellas acciones que parecen considerarse por afuera del control institucional de la religión: las "limpias" con yerberos, curanderos, brujos, santeros, espiritistas o chamanes; la "carga de energía" en "lugares sagrados" como pirámides o santuarios naturales; la práctica de "yoga", meditación u otra "técnica espiritual oriental"; y los rituales indígenas, "danzas" o baños de "temazcal".

La continuidad o presencia de estas prácticas "alternativas" así como de otras dentro del universo religioso no debe considerarse, sin embargo, una ruta hacia la conformación de un esquema religioso o espiritual. En nuestro trabajo cualitativo con desafiliados en la Ciudad de México, los hallazgos han mostrado que:

"la asistencia a servicios religiosos o la congregación esporádica no representa una contradicción en términos de la falta de identificación con un linaje creyente, puesto que los desafiliados subrayan constantemente los arreglos a fines y el carácter voluntarista de sus acciones. Así pues, las personas sin religión suelen realizar acciones del tipo religioso, por ejemplo, para evitar la tensión en la socialización con el círculo familiar inmediato o con los pares; 
para enriquecerse de los valores culturales que se reconocen en los discursos religiosos o espirituales; o para beneficiarse de los efectos terapéuticos y anímicos de ciertas prácticas que han surgido sobre todo en el contexto urbano mexicano en los últimos años, como el yoga, los rituales étnicos o las variantes neotradicionalistas" (Mora, 2017, pp. 263-264).

En resumen, se puede decir que menos de la mitad de las personas sin religión en México conservan un compendio de creencias y prácticas religiosas asociados a los linajes creyentes del campo religioso. Este fenómeno solo se presenta entre el conjunto de desafiliados ya que habitualmente aquellos que se identifican como afiliados o pertenecientes a una religión conservan representaciones sustantivas como la creencia en Dios y la asistencia a servicios religiosos.

En términos de las prácticas, los sin religión se caracterizan mucho más como no asistentes a prácticas religiosas que como no creyentes. Como se pudo observar, mientras 8 de cada 10 nunca asiste a servicios religiosos, alrededor de 4 de cada 10 aún afirma su creencia en Dios, aunque, esta actitud está lejos de ser vinculante con un linaje creyente particular.

Considerando los matices mostrados, es prudente dar cuenta de la posible composición de los perfiles de las personas sin religión en México a partir de su propia identificación, es decir, una vez que las personas se han reconocido fuera de cualquier iglesia o linaje religioso.

\section{Antecedentes y componentes internos de los desafiliados}

Una de los puntos importantes, una vez que se ha identificado la magnitud del número de personas sin religión es observar, por un lado, el antecedente de esta posición de desafiliación religiosa $\mathrm{y}$, segundo, la configuración de las razones principales para que las personas se excluyan de alguno de los linajes creyentes.

En este sentido, se ha encontrado, que la categoría sin religión es la opción con mayor incremento respecto a la identificación con la cual crecen los individuos en el campo religioso mexicano. Según los datos del Pew Research Center, el catolicismo pierde $9 \%$ entre la afiliación de crecimiento y la actual. Por su parte, el sector protestante aumenta $4 \%$, otras religiones suman $2 \%$ y los desafiliados adhieren $5 \%$ (Gráfica 5). 
Gráfica 5. Porcentaje de afiliación religiosa de crecimiento y actual en México, 2014

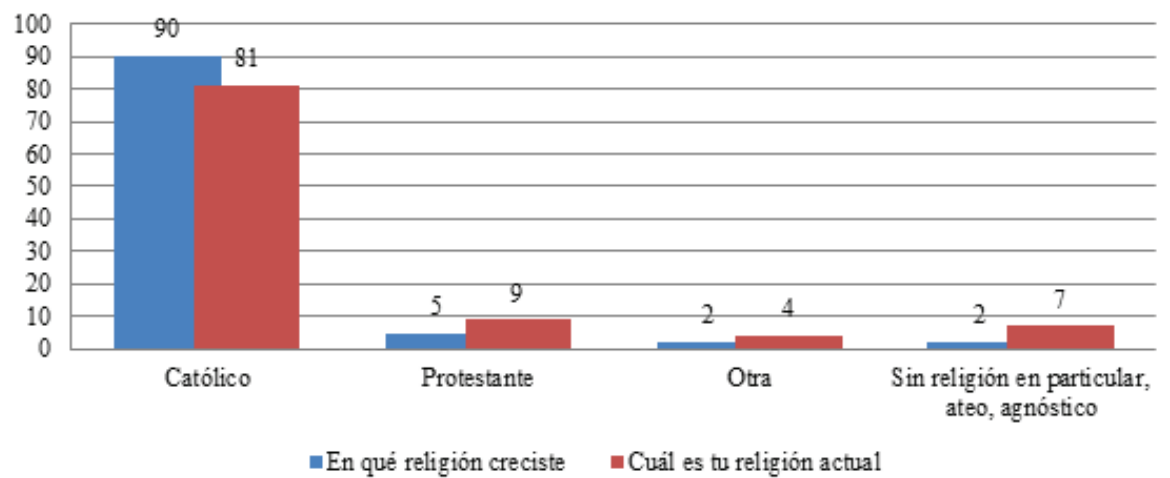

Elaboración propia. Fuente: Pew Research Center (2014).

Este cambio porcentual equivale a decir que el catolicismo tiene una disminución de $10 \%$ de su proporción inicial. Los protestantes incremen$\tan 80 \%$, otras religiones aumentan $100 \% \mathrm{y}$, por su parte, las personas sin religión muestran un cambio sustancial de $250 \%$.

Debido a que el catolicismo es el único grupo que decrece en términos porcentuales, es lógico pensar que hay una transferencia de éste hacia los otros conjuntos del campo religioso, sobre todo a los sin religión. Esta conjetura es confirmada por los datos de la ENCREER (RIFREM, 2016), donde se observa que $75.3 \%$ de las personas que no pertenecen a una religión afirma también que su "anterior religión o culto" era el catolicismo. A esto hay que agregar que, en el momento de definir su identificación actual (“¿Cómo se identifica usted principalmente en términos religiosos en la actualidad?”), casi 7 de cada 10 personas sin pertenencia religiosa $(69.4 \%$ ) se definió mucho más como indiferente, espiritual pero sin iglesia, no practicante, ateo o agnóstico. Y solo aproximadamente 1 de cada 4 (24.8\%) comentó percibirse como creyente (por tradición, convicción, practicante o a su manera).

Más allá de concentrar la atención en las identificaciones diversas de la población sin religión en México, en este artículo se conjetura una tipología destacando las principales justificaciones o razones que esgrimen los desafiliados para posicionarse de esta manera. Para tal propósito, se utilizan los indicadores de la pregunta "si no pertenece a alguna religión, ¿podría indicar la razón?”. Este cuestionamiento es uno de los aportes más importantes de 
la ENCREER (RIFREM, 2016), para profundizar en el conocimiento del sector sin religión en México (Gráfica 6).

Gráfica 6. Porcentaje entre las razones para no pertenecer a alguna religión en México, 2014

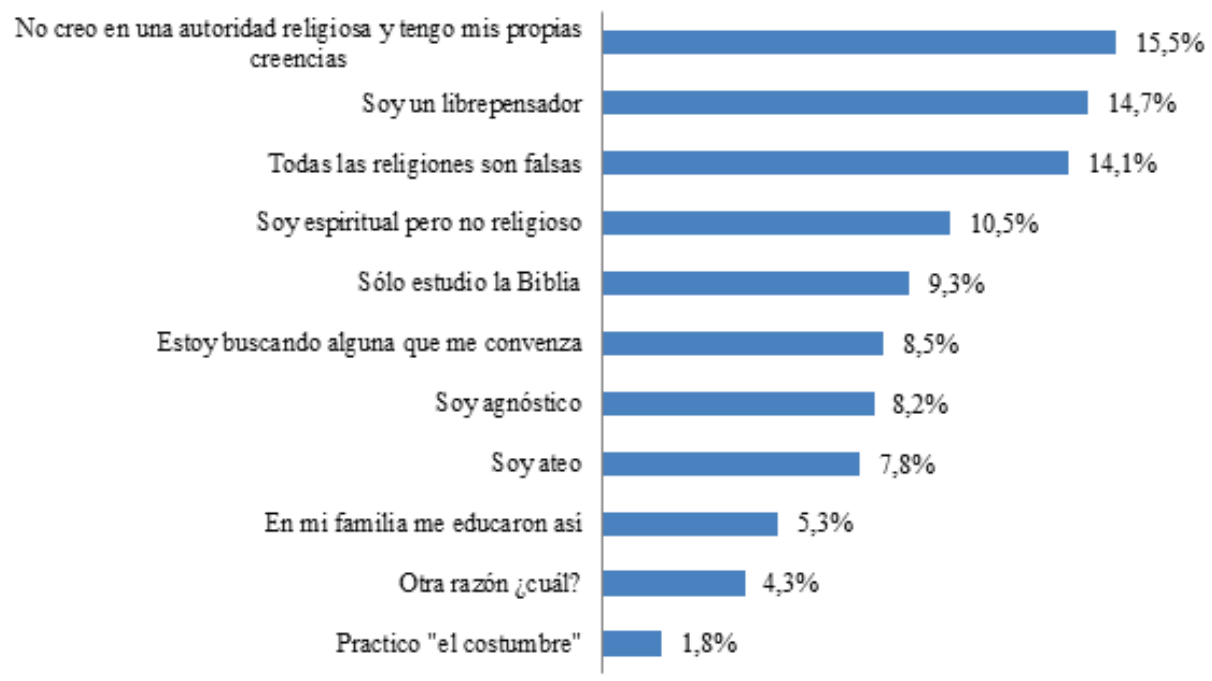

Elaboración propia. Fuente: ENCREER (RIFREM, 2016).

En la gráfica anterior se visualizan las distintas razones para no pertenecer a una religión entre los mexicanos desafiliados. Se presentan 11 distintas, sin embargo, destaca que las primeras cinco representan $64,1 \%$ de todos las personas sin religión. Esto es, que casi 2 de cada 3 desafiliados refiere no pertenecer a una iglesia debido a que "no cree en una autoridad religiosa...", es un "libre pensador", considera que "todas las religiones son falsas", se percibe como "espiritual pero no religioso" o solo "estudia la biblia" por su cuenta.

Una segunda observación a partir de los resultados de la encuesta es el reconocimiento de ciertos grupos que se han supuesto como parte nuclear de las personas sin religión, pero de los cuales no se conoce con exactitud su proporción exacta: los ateos, los agnósticos y las personas de "costumbre". En estos términos, solo $16 \%$ se considera agnóstico o ateo, mientras que la práctica de la costumbre -característica entre comunidades rurales e indígenas de México- solo es representativa para menos del $2 \%$ de los individuos en 
la categoría sin religión. Considerando lo anterior, se puede afirmar que una definición que comprenda solo a ateos, agnósticos o personas de costumbre estaría dando cuenta exclusivamente de $17.8 \%$ del total de personas que no se identifica como miembro de una institución religiosa.

Ahora bien, analizando las consonancias de las diversas razones para justificar la desafiliación religiosa se propone una tipología con tres actitudes confluyentes: la crítica, la autonomía y la indiferencia (Tabla 4).

Tabla 4. Porcentaje de actitudes de personas que no pertenecen a una religión en México, 2014

\begin{tabular}{|l|r|}
\hline Actitud & Porcentaje \\
\hline Crítica & $\mathbf{5 2 . 1 \%}$ \\
\hline $\begin{array}{l}\text { No creo en una autoridad religiosa y tengo mis } \\
\text { propias creencias }\end{array}$ & $15.5 \%$ \\
\hline Soy un librepensador & $14.7 \%$ \\
\hline Todas las religiones son falsas & $14.1 \%$ \\
\hline Soy ateo & $7.8 \%$ \\
\hline Autonomía & $\mathbf{2 8 . 3 \%}$ \\
\hline Soy espiritual pero no religioso & $10.5 \%$ \\
\hline Sólo estudio la Biblia & $9.3 \%$ \\
\hline Estoy buscando alguna que me convenza & $8.5 \%$ \\
\hline Indiferencia & $\mathbf{1 5 . 3} \%$ \\
\hline Soy agnóstico & $8.2 \%$ \\
\hline En mi familia me educaron así & $5.3 \%$ \\
\hline Practico “el costumbre” & $1.8 \%$ \\
\hline Otro & $\mathbf{4 . 3 \%}$ \\
\hline Total & $\mathbf{1 0 0} \%$ \\
\hline
\end{tabular}

Fuente: ENCPRM, 2017. Nota: Se excluyó de la categorización la respuesta “Otra razón”.

De acuerdo con la tipología propuesta en la tabla anterior, alrededor de 1 de cada 2 personas que no pertenecen a una religión (52.1\%) expresa una actitud crítica hacia la cuestión religiosa. Está contraposición se expresa en el argumento de que "todas las religiones son falsas" y no se "cree en una 
autoridad". Y a esto se suma la definición de los "ateos" y de los "librepensadores" quienes cuestionan, por un lado, la probabilidad de la existencia de una entidad superior (Dios, creador, sustancia divina, etc.) o que aseguran que la religión limita la libertad de pensamiento. ${ }^{5}$

Por otro lado, la autonomía es un segundo comportamiento entre aproximadamente una tercera parte de las personas sin religión. Esta actitud involucra la reafirmación de la agencia individual al disponer y reorganizar los recursos de las tradiciones religiosas conjuntamente con discursos de otras fuentes e ideologías. Con base en lo anterior, generalmente el autónomo afirma, en primer término, ser "espiritual pero no religioso", aunque también puede "estudiar la biblia" o estar en "búsqueda" de algún linaje religioso que le convenza. Esta reafirmación individual, además, conlleva a una pérdida del predominio de los sistemas religiosos sobre la experiencia de las personas, no como consecuencia de un cuestionamiento intrínseco, sino por el pleno ejercicio de la capacidad de una sociedad para "orientar por sí misma su destino, y la recomposición, bajo una nueva forma, de las representaciones religiosas que han permitido a esta sociedad pensarse a sí misma como autónoma" (Hervieu-Léger, 1999, p. 37).

Finalmente, la tercera actitud, entre $15.3 \%$ de las personas sin pertenencia religiosa, puede ser definida como indiferencia o, incluso, como una posición acrítica respecto a los elementos religiosos. En este subcojunto se aglutinan a aquellos que practican "la costumbre", y aquellos otros que aseguraron haber sido educados "así" en la familia, es decir, con una probable experiencia de "educación secular" o de "religiosidad laxa" que les permitió alejarse progresivamente del paraguas de la religión. ${ }^{6}$ A lo anterior, se agrega, igualmente, la posición de los "agnósticos" o, en otras palabras, aquellos que no niegan la existencia de lo trascendente como los ateos, pero "no le otorgan ningún sentido" o "afirman su incognoscibilidad racional" (Bericat,

5 Sobre el caso de los librepensadores, se coincide con los hallazgos de Keysar y Kosmin (2007), quienes encuadran a estos individuos dentro del subgrupo de aquellos sin religión que han rechazado conscientemente a la religión y, en tal caso, "un comportamiento claro que identifica a un librepensador es la apostasía o la disposición a renunciar a una identidad religiosa previamente celebrada" (Kosmin \& Keysar, 2007, p. 20).

6 A través del análisis cualitativo de las trayectorias de vida de personas sin religión se ha encontrado perfiles de personas con una historia biográfica de "religión laxa" y, en menores porcentajes, con una experiencia de educación y crecimiento sin elementos formales de una tradición religiosa, a quienes se ha denominado como "nativos seculares" (Mora, 2017, p. 211). 
2008). También entre estos últimos, su posicionamiento puede comprender un componente de indiferencia o apatía más que de alejamiento o crítica.

\section{A manera de conclusión}

Se ha planteado en este artículo la descripción de las principales características de la población sin religión en México. A través de la investigación de los datos de encuestas y del censo nacional mexicano, parece sostenerse el planteamiento de la reorganización permanente del fenómeno religioso supuesto por la secularización. Asimismo, se aporta información para sustentar que la secularización de la conciencia o individual comprende perfiles y actitudes distintas, desde el movimiento continuo entre la afiliación, las creencias y las prácticas religiosas, hasta la propia diversificación de actitudes respecto a la cuestión religiosa.

Bajo esta lógica, se ha observado que las personas sin religión en México son un sector joven de la población con menos concurrencia hacia las creencias y prácticas de los linajes creyentes, sin embargo, no se puede afirmar que estos individuos han descartado completamente los recursos que beredaron de su afiliación religiosa. En algunos casos, su apropiación configura actitudes de crítica hacia los dogmas e instituciones religiosas; en otros, se desarrolla la gestión individual de los bienes de salvación bajo la tutela de la autenticidad y de la plenitud humana; y, sin duda, en algunos otros, se adquiere una actitud de irrelevancia o de "indiferencia creyente"; esto es, una de las tantas posibilidades atribuidas a las condiciones de posibilidad de la secularización (Hervieu-Léger, 1999).

\section{Bibliografía}

Bastian, J.-P. (2008). Conversiones religiosas y redefinición de la etnicidad en el estado de Chiapas. Trace [En línea], 19-30.

Bastian, J.-P. (2013). Pluralización religiosa y lógica de mercado en América Latina. En M. A. Cuadriello \& M. T. Kuri, El fin de un sueño secular: religión y relaciones internacionales en el cambio de siglo (Primera edición, pp. 91-112). México: El Colegio de México.

Bericat, E. (2008). Duda y posmodernidad: el ocaso de la secularización en Europa (Doubt and Postmodernity: The Decline of Secularisation in Europe). Reis, (121), 13-53. https:// doi.org/10.2307/40184844

Católicas por el Derecho a Decidir. (2014). Encuesta Nacional de Opinión Católica 2014 (En línea). México: Católicas por el Derecho a Decidir. Recuperado a partir de http://encuesta. catolicasmexico.org/es/ 
Condran, J. G., \& Tamney, J. B. (1985). Religious “nones”: 1957 to 1982. Sociology of Religion, 46(4), 415-423.

de la Torre, R., \& Gutiérrez, C. (2007). Atlas de la diversidad religiosa en México. México: El Colegio de Jalisco. Recuperado a partir de http://books.google.com.mx/books?id=E0V-E_FTjtMC

Gibaja, R. E. (1972). Religión y Secularización entre campesinos y obreros. Revista Mexicana de Sociología, 34(2), 193-244. https://doi.org/10.2307/3539506

Gutiérrez, C. (2007). Población sin religión. En R. de la Torre \& C. Gutiérrez, Atlas de la diversidad religiosa en México (pp. 116-123). México: El Colegio de Jalisco.

Hervieu-Léger, D. (1985). Secularización y modernidad religiosa. Esprit, 106, 50-62.

Hervieu-Léger, D. (1996). Por una sociología de las nuevas formas de religiosidad: algunas cuestiones teóricas previas. En Identidades Religiosas y Sociales en México (Primera edición, pp. 23-46). México: Instituto de Investigaciones Sociales, Universidad Nacional Autónoma de México.

Hervieu-Léger, D. (1999). El peregrino y el convertido: la religión en movimiento. México: Ediciones del Helénico.

INEGI. (2005). La diversidad religiosa en México. XII Censo de Población y Vivienda 2000 (p. 192). México: Instituto Nacional de Estadística y Geografía. Recuperado a partir de http://www. inegi.org.mx/prod_serv/contenidos/espanol/bvinegi/productos/integracion/sociodemografico/religion/div_rel.pdf

INEGI. (2010). Censo de Población y Vivienda 2010. Instituto Nacional de Estadística y Geografía. Recuperado a partir de http://www.censo2010.org.mx/

INEGI. (2011). Panorama de las religiones en México 2010 (p. 270 págs.). México: Instituto Nacional de Estadísitca y Geografía. Recuperado a partir de http://www.inegi.org.mx/ prod_serv/contenidos/espanol/bvinegi/productos/censos/poblacion/2010/panora_religion/ religiones_2010.pdf

INEGI. (2015). Clasificación de religiones 2010. Instituto Nacional de Estadística y Geografía. Recuperado a partir de http://internet.contenidos.inegi.org.mx/contenidos/productos/ prod_serv/contenidos/espanol/bvinegi/productos/nueva_estruc/702825064983.pdf

Kosmin, B. A., \& Keysar, A. (2007). The Freethinkers in a Free Market of Religion. En Secularism \& Secularity: Contemporary International Perspectives (pp. 17-26). Institute for the Study of Secularism in Society and Culture.

Lafaye, J. (2015). Quetralcóatl y Guadalupe: La formación de la conciencia nacional en México. Abismo de conceptos. Identidad, nación, mexicano. Fondo de cultura económica.

Latinobarómetro. (2014). Las religiones en tiempos del Papa Francisco (p. 34). Santiago de Chile: Latinobarómetro. Recuperado a partir de www.latinobarometro.org

LeDrew, S. (2013). Discovering Atheism: Heterogeneity in Trajectories to Atheist Identity and Activism. Sociology of Religion. https://doi.org/10.1093/socrel/srt014

LeDrew, S. (2014, marzo 21). Atheists are Believers [Blog]. Recuperado el 15 de septiembre de 2014, a partir de http://blog.nsrn.net/2014/03/21/atheists-are-believers/

Mora, C. (2017, enero 24). Creer sin iglesia y practicar sin Dios: población sin religión en el contexto 
urbano y rural de México en los albores del Siglo XXI. El Colegio de México, Ciudad de México. Recuperado a partir de http://ces.colmex.mx/pdfs/tesis/tesis_mora_duro.pdf

Parametría. (2015). Encuesta Nacional en vivienda (En línea). Ciudad de México: Parametría. Recuperado a partir de http://www.parametria.com.mx/carta_parametrica.php?cp $=4827$

Pew Research Center. (2014). Religión en América Latina. Cambio generalizado en una región históricamente católica (p. 28). Estados Unidos: Pew Research Center's Religion \& Public Life Project. Recuperado a partir de http://www.pewforum.org/files/2014/11/PEW-RESEARCH-CENTER-Religion-in-Latin-America-Overview-SPANISH-TRANSLATION-for-publication-11-12-1.pdf

RIFREM. (2016). Encuesta Nacional sobre Creencias y Prácticas Religiosas en México (Estadístico). México: Red de Investigadores del Fenómeno Religiosos en México.

Smith, J. M. (2010). Becoming an Atheist in America: Constructing Identity and Meaning from the Rejection of Theism. Sociology of Religion. https://doi.org/10.1093/socrel/srq082

Trzebiatowska, M., \& Bruce, S. (2012). Why are Women More Religious Than Men? OUP Oxford. 